\title{
BMJ Open Prevalence of diabetic retinopathy in children and young people living with diabetes: protocol for a systematic review
}

\author{
Maria Carolina Ibanez-Bruron, ${ }^{1,2}$ Ameenat L Solebo, ${ }^{1,2,3,4,5}$ \\ Phillippa M Cumberland, , ${ }^{1,2}$ Jugnoo S Rahi ${ }^{1,2,3,4,5}$
}

To cite: Ibanez-Bruron MC, Solebo AL, Cumberland PM, et al. Prevalence of diabetic retinopathy in children and young people living with diabetes: protocol for a systematic review. BMJ Open 2017;7:e018578. doi:10.1136/ bmjopen-2017-018578

- Prepublication history and additional material for this paper are available online. To view these files, please visit the journal online (http://dx.doi. org/10.1136/bmjopen-2017018578).

Received 7 July 2017 Revised 12 0ctober 2017 Accepted 13 October 2017

CrossMark

${ }^{1}$ GOS Institute of Child Health, University College London, London, UK

${ }^{2}$ Ulverscroft Vision Research Group, London, UK

${ }^{3}$ Great Ormond Street Hospital for Children NHS Foundation

Trust, London, UK

${ }^{4}$ Moorfields Eye Hospital NHS Foundation Trust, London, UK ${ }^{5}$ Institute of Ophthalmology, University College London, London, UK

Correspondence to Professor Jugnoo S Rahi; j.rahi@ucl.ac.uk

\section{ABSTRACT}

Introduction The frequency of diabetes mellitus in childhood is increasing. Thus, more children and young people are at risk of developing diabetic retinopathy and diabetes related visual impairment. However, there is no consensus on optimal screening strategies for the paediatric population reflecting the lack of clarity about the current burden of disease in this group. We aim to estimate the prevalence of diabetic retinopathy in children and young people living with types 1 or 2 diabetes, and to investigate potential sources of heterogeneity in this figure so as to inform screening strategies for this population. Methods and analysis PubMed and EMBASE will be searched from 1995 to 2016 using the OvidSP platform with no language restriction. Additionally, manual review of the references lists of included articles will be conducted. Two investigators will independently screen titles and abstracts for potential eligibility. Studies which report prevalence of diabetic retinopathy among general populations of children and young people with types 1 or 2 diabetes will be included. Pooled prevalence estimates of diabetic retinopathy reported in studies with sample size greater than 200 participants will be calculated by the random effect model. Forest plots will be used to summarise individual and pooled estimates of the prevalence. Heterogeneity between studies will be assessed using the $P^{2}$ statistic and explored through metaregressions and subgroup analyses if the necessary data are available.

Ethics and dissemination Ethics approval is not required as this is a review of anonymised published data. We will report the findings of this systematic review in a peer-reviewed journal, and share it with the relevant professionals including health authorities through our Diabetic Eye disease in Childhood Study collaborative network.

Clinical trail registration PROSPERO (CRD42017067178).

\section{BACKGROUND}

Diabetic retinopathy is the most common preventable cause of visual impairment in young adults. ${ }^{1}$ The rising prevalence of childhood type 1 and type 2 diabetes $^{2-4}$ has led to
Strengths and limitations of this study

- This formal systematic review will provide currently unavailable information on the prevalence of diabetic retinopathy in the paediatric population.

- Only studies reporting diagnostic methods and grades of diabetic retinopathy will be included to enhance precision.

- To increase the applicability of the findings, two severity cut-offs will be used to calculate prevalence estimates.

- As with all systematic reviews, the poor reporting of methods in primary studies might compromise the exploration of the heterogeneity.

a significant increase in the number of children and young people at risk of visual loss.

Microvascular retinal damage is a marker of general vascular health, indicative of a need for greater diabetic disease control. The more advanced, but still asymptomatic, stages of diabetic retinopathy require additional ophthalmic intervention to reduce the risk of irreversible visual loss. Screening for diabetic retinopathy in children and young people is recommended by many national population health programmes, with commencement of annual retinal examination determined either by age (typically age 10-12 years) or duration of disease (typically $2-5$ years from diagnosis) ${ }^{5-7}$

Although it has been suggested that these recommendations need to be revised based on recent evidence on the burden of the disease in this population, there is no consensus on the direction of these modifications. ${ }^{8-12}$ Variable estimates of the prevalence of diabetic retinopathy may be due in part to heterogeneity between study populations, diagnostic methods or definitions of diabetic retinopathy used in the studies. ${ }^{13} 14$ Consensus about screening strategies for the 
paediatric population required greater clarity in evidence base regarding the natural history and the burden of disease in this group. ${ }^{15-17}$

The aim of this systematic review is to estimate the prevalence of diabetic retinopathy in children and young people living with type 1 or type 2 diabetes, and to investigate potential sources of heterogeneity such as study sample characteristics (eg, age, diabetes type and duration, and glycaemic control), diagnostic methods and definition of diabetic retinopathy to inform screening strategies for the paediatric population.

\section{METHODS/DESIGN}

The systematic review will be performed in accordance with the Preferred Reporting Items for Systematic Reviews and Meta-Analyses (PRISMA) guidelines, with this protocol written according to the PRISMA-P checklist. ${ }^{18}$ The PRISMA-P checklist can be found as online supplementary additional file 1 . The study has been registered on PROSPERO (CRD42017067178).

\section{Data sources and searches}

The PubMed and EMBASE databases will be searched using the OvidSP platform. We will also manually review the references lists of identified articles. The search strategy (table 1) will include terms related to children or young people, diabetes, diabetic retinopathy, prevalence and diagnostic tests, and there will be no language restrictions. We will exclude studies published before January 1995 as older studies are likely to have been conducted prior to the publication of the Diabetes Control and Complications Trial, which demonstrated decreased risk of diabetic retinopathy development or progression with tighter glycaemic control than that previously used in routine clinical practice, and may have led to significant changes in disease management and natural history. ${ }^{19}$

\section{Study eligibility criteria}

We will include studies which report prevalence estimates of diabetic retinopathy among general populations of children and young people with diabetes mellitus, or studies from which the prevalence data may be derived (table 2). Eligible studies will be those which meet the following criteria.

- Participants

Individuals aged under 18 years living with types 1 or 2 diabetes. Studies with median/mean age of the sample greater than 25 years or which include participants older than 30 years will be excluded.

- Disease measure

Report of the clinical examination method used to determine diabetic retinopathy status, and description of either retinal findings or grade of retinopathy using any grading systems which can be correlated to retinopathy features seen at the ophthalmic examination. This will allow comparison between the different grading systems used in screening programmes and
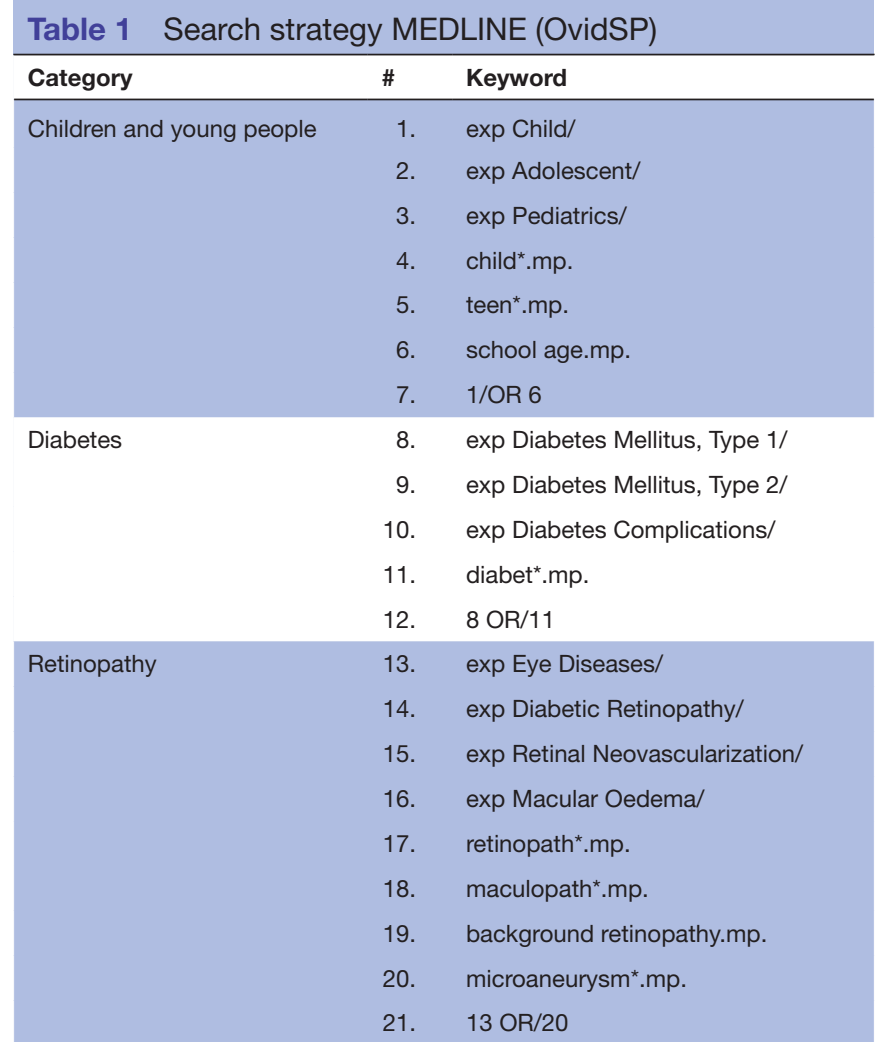

Prevalence

13. exp Eye Diseases/

14. exp Diabetic Retinopathy/

15. exp Retinal Neovascularization/

16. exp Macular Oedema/

17. retinopath ${ }^{\star} . \mathrm{mp}$.

18. maculopath*.mp.

19. background retinopathy.mp.

20. microaneurysm.mp.

21. $13 \mathrm{OR} / 20$

22. exp Epidemiology/

23. exp Prevalence/

24. exp Incidence/

25. exp Natural History/

26. frequenc ${ }^{\star} \cdot \mathrm{mp}$.

27. epidemiolog*.mp.

28. prevalen ${ }^{\star} . \mathrm{mp}$.

29. inciden*.mp.

30. severity.mp.

31. $22 \mathrm{OR} / 30$

$\begin{array}{lll}\text { Diagnostic tests } & \text { exp Photography/ } \\ 33 . & \text { exp Ophthalmoscopes/ } \\ 34 . & \text { exp Fluorescein Angiography/ } \\ 35 . & \text { exp Fundus Oculi/ } \\ 36 . & \text { exp Slit Lamp/ } \\ 37 . & \text { exp ophthalmoscopy/ } \\ 38 . & \text { Tomography, Optical Coherence/ } \\ 39 . & \text { Ultrasonography/ } \\ 40 . & \text { photo*mp. } \\ 41 . & \text { ophthalmoscop*. mp. } \\ 42 . & \text { angiogram*. mp. } \\ 43 . & \text { biomicroscopy.mp. } \\ 44 . & \text { Optical coherence tomography.mp. } \\ 45 . & \text { ultrasonograph*.mp. } \\ 46 . & \text { fundus.mp. } \\ 47 . & \text { Slim Lamp.mp. } \\ 48 . & \text { fundoscop*.mp. } \\ 49 . & \text { 32 OR/48 } \\ 50 . & 7 \text { AND } 12 \text { AND } 21 \text { AND } 31 \text { AND } 49 \\ 51 . & \text { limit } 50 \text { to yr='1995 -Current' } \\ & \end{array}$


Table 2 Inclusion and exclusion criteria

\begin{tabular}{|c|c|c|}
\hline Category & Inclusion criteria & Exclusion criteria \\
\hline Population & $\begin{array}{l}\text { Children and young people <18years old included in } \\
\text { the sample } \\
\text { Children and young people from general population }\end{array}$ & $\begin{array}{l}\text { Median/mean sample age }>25 \text { years } \\
\text { Participants }>30 \text { years old included } \\
\text { Studies conducted in selected populations (eg, } \\
\text { dialysis, post-transplanted) }\end{array}$ \\
\hline Exposure & - Type 1 or 2 diabetes & - Other types of diabetes (eg, monogenic causes) \\
\hline $\begin{array}{l}\text { Outcome of } \\
\text { interest }\end{array}$ & $\begin{array}{l}\text { Prevalence of diabetic retinopathy } \\
\text { Test used to assess diabetic retinopathy reported }\end{array}$ & $\begin{array}{l}\text { Estimation of prevalence not possible with } \\
\text { published data }\end{array}$ \\
\hline Article & $\begin{array}{l}\text { Published between } 1 \text { January } 1995 \text { and } 31 \text { October } \\
2016\end{array}$ & $\begin{array}{l}\text { Duplicate reports (the most comprehensive } \\
\text { version will be included) }\end{array}$ \\
\hline
\end{tabular}

clinical practice. Included grading systems will be displayed in a supplementary table along with the systematic review's results.

Prevalence will be calculated using two cut-offs, any diabetic retinopathy and sight-threatening diabetic retinopathy, that is, grades of retinopathy that require prompt ophthalmic management to reduce the risk of irreversible visual impairment. Any diabetic retinopathy will be defined as background retinopathy or more severe disease. Sight-threatening diabetic retinopathy $^{20}$ will be primarily defined as proliferative diabetic retinopathy or clinically significant maculopathy oedema, that is, retinal thickening at or within $500 \mu \mathrm{m}$ of the centre of the macula, and/or hard exudates at or within $500 \mu \mathrm{m}$ of the centre of the macula, if associated with thickening of the adjacent retina, and/or a zone or zones of retinal thickening one disc area in size, any part of which is within one disc diameter of the centre of the macula. ${ }^{21}$ Additionally, a secondary analysis using a more inclusive definition of sight-threatening diabetic retinopathy, that is, including severe non-proliferative diabetic retinopathy, will be conducted using the available data.

Conference abstracts with sufficient data on disease measure to determine eligibility will be included.

\section{Study selection}

The search results will be extracted and managed using Microsoft Excel (Microsoft Corporation, Redmond, Washington, USA). Two independent investigators (MIB, ALS) will screen titles and abstracts, and then apply the eligibility criteria to full-text articles. Disagreements will be resolved by consensus following discussion. Arbitration by a third author (JSR) will be sought whenever necessary.

\section{Data extraction}

Two investigators (MIB, ALS) will extract the data using a standardised form. The following data items will be extracted: first author, year of publication, design, country, year of study conduction, sample size, diabetes type, age at examination, diabetes duration, HbAlc, diagnostic test, diabetic retinopathy definition, number of any diabetic retinopathy cases, severity of diabetic retinopathy cases. If a study reports multiple prevalence estimates over time, the first prevalence estimate, that is, the estimate which is reported with baseline sample clinical characteristics, will be selected as the most informative measure. Characteristics of children and young people with sight-threatening diabetic retinopathy will be also extracted. Authors will be contacted if data in the original publication is unclear.

\section{Assessment of risk of bias in included studies}

The risk of bias will be assessed with an adapted version of the tool developed by Hoy and colleagues ${ }^{22}$ (online Supplementary additional file 2). The sample will be considered representative of the target population (ie, 'general' population) when all children and young people registered in a paediatric diabetes clinic, or living in a specified catchment area were potentially eligible for the study. Sources of information will be classified as appropriate where diabetic retinopathy status is assessed during the study (cross-sectional and prospective studies) or where prospective databases such as diabetic retinopathy screening registers are used.

\section{Data synthesis}

A comprehensive table for summary of findings with narrative description of all eligible studies will be reported. However, only studies with a sample size equal or greater than 200 participants will be included in the meta-analysis. Small studies will be excluded to reduce variability due to imprecise estimates. Pooled prevalence estimates of any diabetic retinopathy and of sight-threatening diabetic retinopathy will be calculated by random effect model meta-analysis. Forest plots will be used to summarise individual and pooled estimates of the prevalence. Heterogeneity between studies will be assessed using the $I^{2}$ statistic (values of $25 \%, 50 \%$ and $75 \%$ will be considered low, medium and high heterogeneity, respectively) ${ }^{23}$ and will be further explored through meta-regressions and subgroup analyses considering the following covariates: sample median/mean age, median/mean diabetes duration, type of diabetes, median/mean HbA1c, diagnostic test, and year of study conduction. All analyses will be performed with $R$ statistical software V.3.3.2., and $\mathrm{p}$ values $<0.05$ will 
be used as the threshold for statistical significance. Assessment of meta-bias(es) is not planned, as preliminary searches of registered protocols have indicated that it is unlikely that eligible epidemiological studies are prospectively registered. As this systematic review is not investigating the evidence underpinning an intervention, confidence in the cumulative evidence cannot be calculated or assessed using the Grading of Recommendations Assessment, Development and Evaluation. The strength of the body of evidence will be examined through the assessment of risk of bias within included studies.

\section{DISCUSSION}

Diabetic retinopathy is, in the early stages, an important marker of disease control, and in the later stages, a preventable cause of visual impairment and blindness. The burden of childhood diabetes is increasing, but there is a paucity of synthesised data on the prevalence and natural history of childhood diabetic retinopathy. This systematic review, using a clinically impactful taxonomy to calculate the prevalence of diabetic retinopathy, will provide currently unavailable prevalence estimates to inform screening policy and practice for children and young people living with diabetes.

\section{Ethics and dissemination}

Ethical approval is not needed as this study will use only anonymised published data. We will report the findings of this systematic review in a peer-reviewed journal, and share it with the relevant professionals including health service planners through our DECS collaborative network.

Contributors JSR is the guarantor. MCIB designed the study and drafted the protocol. ALS, PMC and JSR critically revised and provided feedback on the protocol. MCIB, ALS, PMC and JSR read and approved the final version of the manuscript.

Funding This work was funded by the Ulverscroft Foundation, the National Institute for Health Research (NIHR) Biomedical Research Centre at University College London Institute of Child Health/Great Ormond Street Hospital NHS Foundation Trust, Diabetes Research \& Wellness Foundation and the National Commission for Scientific and Technological Research in Chile (CONICYT). ALS and JR are also funded by the NIHR Moorfields Biomedical Research Centre. The funding organisations had no role in the design or conduct of this research.

\section{Competing interests None declared.}

Provenance and peer review Not commissioned; externally peer reviewed.

Open Access This is an Open Access article distributed in accordance with the terms of the Creative Commons Attribution (CC BY 4.0) license, which permits others to distribute, remix, adapt and build upon this work, for commercial use, provided the original work is properly cited. See: http://creativecommons.org/ licenses/by/4.0/

(c) Article author(s) (or their employer(s) unless otherwise stated in the text of the article) 2017. All rights reserved. No commercial use is permitted unless otherwise expressly granted.

\section{REFERENCES}

1. Liew G, Michaelides M, Bunce C. A comparison of the causes of blindness certifications in England and Wales in working age adults (16-64 years), 1999-2000 with 2009-2010. BMJ Open 2014;4:e004015.

2. Haines L, Wan KC, Lynn R, et al. Rising incidence of type 2 diabetes in children in the U.K. Diabetes Care 2007;30:1097-101.

3. Patterson CC, Dahlquist GG, Gyürüs E, et al. Incidence trends for childhood type 1 diabetes in Europe during 1989-2003 and predicted new cases 2005-20: a multicentre prospective registration study. Lancet 2009;373:2027-33.

4. Dabelea D, Mayer-Davis EJ, Saydah S, et al. SEARCH for Diabetes in Youth Study. Prevalence of type 1 and type 2 diabetes among children and adolescents from 2001 to 2009. JAMA 2014;311:1778-86.

5. American Diabetes Association. Microvascularcomplications and foot care. Sec. 9. In Standards of Medical Care inDiabetes-2016. Diabetes Care 2016;39:S72-80.

6. Donaghue KC, Wadwa RP, Dimeglio LA, et al. Microvascular and macrovascular complications in children and adolescents. Pediatr Diabetes 2014;15:257-69.

7. National Institute for Health and Care Excellence (NICE). Diabetes (type 1 and type 2) in children and young people: diagnosis and management. 2015 https://www.nice.org.uk/guidance/ng18 (accessed Oct 2017).

8. Beauchamp G, Boyle CT, Tamborlane WV, et al. Treatable Diabetic Retinopathy Is Extremely Rare Among Pediatric T1D Exchange Clinic Registry Participants. Diabetes Care 2016;39:e218-9.

9. Geloneck MM, Forbes BJ, Shaffer J, et al. Ocular complications in children with diabetes mellitus. Ophthalmology 2015;122:2457-64.

10. Hamid A, Wharton HM, Mills A, et al. Diagnosis of retinopathy in children younger than 12 years of age: implications for the diabetic eye screening guidelines in the UK. Eye 2016;30:949-51.

11. Wang SY, Andrews CA, Herman WH, et al. Incidence and risk factors for developing diabetic retinopathy among youths with type 1 or type 2 diabetes throughout the united states. Ophthalmology 2017;124:424-30.

12. Dabelea D, Stafford JM, Mayer-Davis EJ, et al. Association of type 1 diabetes vs type 2 diabetes diagnosed during childhood and adolescence with complications during teenage years and young adulthood. JAMA 2017;317:825-35.

13. Dinnes J, Deeks J, Kirby J, et al. A methodological review of how heterogeneity has been examined in systematic reviews of diagnostic test accuracy. Health Technol Assess 2005;9:1--113.

14. Ibanez-Bruron MC, Solebo AL, Rahi JS. Complications of diabetes diagnosed in children and adolescents. JAMA 2017;317:2553.

15. Lueder GT, Silverstein J. American Academy of Pediatrics Section on Ophthalmology and Section on Endocrinology. Screening for retinopathy in the pediatric patient with type 1 diabetes mellitus. Pediatrics 2005;116:270-3.

16. Thomas RL, Harvey JN, Owens DR. When should screening for diabetic retinopathy begin for children with type 1 diabetes? Expert Rev Endocrinol Metab 2016;11:97-102.

17. Maguire A, Chan A, Cusumano J, et al. The case for biennial retinopathy screening in children and adolescents. Diabetes Care 2005;28:509-13.

18. Moher D, Shamseer L, Clarke M, et al. Preferred reporting items for systematic review and meta-analysis protocols (PRISMA-P) 2015 statement. Syst Rev 2015;4:1.

19. Klein R, Klein BE, Moss SE, et al. Relationship of hyperglycemia to the long-term incidence and progression of diabetic retinopathy. Arch Intern Med 1994;154:2169-78.

20. Zhang X, Saaddine JB, Chou CF, et al. Prevalence of diabetic retinopathy in the United States, 2005-2008. JAMA 2010;304:649-56.

21. Kinyoun J, Barton F, Fisher M, et al. Detection of diabetic macular edema. Ophthalmoscopy versus photography--early treatment diabetic retinopathy study report number 5 . The etdrs research group. Ophthalmology 1989;96:746-50.

22. Hoy $D$, Brooks $P$, Woolf $A$, et al. Assessing risk of bias in prevalence studies: modification of an existing tool and evidence of interrater agreement. J Clin Epidemiol 2012;65:934-9.

23. Higgins JP, Thompson SG. Quantifying heterogeneity in a metaanalysis. Stat Med 2002;21:1539-58. 